\title{
The evolution of electroluminescence in Ge quantum-dot diodes with the fold number
}

\author{
Y. H. Peng, Chih-Hsiung Hsu, C. H. Kuan, ${ }^{\text {a) }}$ and C. W. Liu \\ Department of Electrical Engineering and Graduate Institute of Electronics Engineering, \\ National Taiwan University, Taipei, Taiwan \\ P. S. Chen and M.-J. Tsai \\ Electronics Research and Service Organization, Industrial Technology Research Institute, Hsinchu 310, \\ Taiwan, Republic of China \\ Y. W. Suen \\ Department of Physics, National Chung Hsing University, Taichung, Taiwan
}

(Received 3 March 2004; accepted 9 November 2004)

\begin{abstract}
The electroluminescence of the light-emitting diodes with five-, ten and 30-fold $p$-type Ge quantum dots grown on $n^{+} \mathrm{Si}$ substrates is studied. The enhanced integral electroluminescence intensity and blueshift of the 30 -fold one at high temperature $(>200 \mathrm{~K})$ act contrary to those in five- and ten-fold ones. It is attributed to the emission in the higher-fold quantum dots enabled by the injected electrons diffusing the farther at the higher temperature. Transmission electron microscopy shows that the size of the Ge quantum dots and the Si component in them, both increase with increasing the fold number. Due to the strain-induced intermixing at the high-fold quantum dots, those dots hence have large band gap and result in the intensity increment and blueshift at the high temperature. (C) 2004 American Institute of Physics. [DOI: 10.1063/1.1842371]
\end{abstract}

The self-assembled Ge quantum dots (QDs) have a promising application for optical communication in 1.3-1.5 $\mu \mathrm{m}$ wavelength. ${ }^{1}$ Those self-assembled QDs form with the strong strain due to the lattice mismatch between materials. Large band offset and strong quantum confinement can enhance the optical characteristics. In the past decade, this feature has attracted many researchers to develop QD optoelectronic detectors ${ }^{2,3}$ and emitters. ${ }^{4-7}$

For emitters, many efforts have been made to enhance the electroluminescence (EL). The best way is to confine the carriers and photons in the active region. The highperformance emitters with the double heterostructure were achieved using $\mathrm{Al}_{x} \mathrm{Ga}_{1-x} \mathrm{As} / \mathrm{GaAs}$ material 30 years ago. However, the $\mathrm{Ge} / \mathrm{Si}$ material system has an indirect band gap. According to the property of the indirect band-gap material, the theoretical recombination probability for the bandto-band transition is so low that it is hard to provide enough radiative efficiency. ${ }^{8}$ By providing the confinement of the carriers, the Ge QDs buried in the Si have strong radiation. ${ }^{9}$ Unfortunately, compared with the III-V material, the Ge/Si material can only provide the confinement for the holes because of its Type II band alignment. In the conventional Ge QD PN diode, the electrons diffuse easily into the $p$-type region by current injection. The built-in field in the depletion region renders the holes hard to capture the electrons. In order to increase the radiative efficiency, many folds of the Ge QD layers are grown in this letter. In addition to the depletion region, the Ge QD layer may exist in the diffusion region of electrons. Compared with the short-fold Ge QD light-emitting diode (LED), unusual intensity increment and blueshift with temperature are found in the spectra of the long-fold one. It is attributed to the increment of the electron diffusion length with temperature.

\footnotetext{
${ }^{\text {a)} E l e c t r o n i c ~ m a i l: ~ k u a n @ c c . e e . n t u . e d u . t w ~}$
}

The Ge QDs with the fold number of 5, 10, and 30 were grown on $n^{+}(100) \mathrm{Si}(0.0018 \Omega \mathrm{cm})$ substrate by a commercial ultrahigh-vacuum chemical vapor deposition system. The Si wafers were cleaned by a dip in HF solution, prior to the deposition of multiple quantum dots (MQDs). The growth temperature was maintained at $600^{\circ} \mathrm{C}$ by vacuum annealing furnace. After 100-nm-thick Si buffer, the five, ten, thirty, $\mathrm{Ge} / \mathrm{Si}$ bilayers were grown including 13.1 equivalentmonolayers (eq-ML, 1 eq-ML $=6.27 \times 10^{14} \mathrm{Ge}$ atoms $/ \mathrm{cm}^{2}$ ) and the $18 \mathrm{~nm}$ Si spacer to reduce the roughness of Ge QDs surface and to form the quantum confinement of the holes. Then, 100-nm-thick undoped Si blocking layer and the top contact layer with boron doping of $1 \times 10^{19} \mathrm{~cm}^{-3}$ were grown on the MQDs. The detail sample structure for our devices was plotted in Fig. 1. The unintentional doping in the undoped region calibrated by secondary ion mass spectroscopy was shown to be about $3 \times 10^{16} \mathrm{~cm}^{-3}$. This background doping will provide enough carriers in the quantum dot samples.

Since the variation of QD size may lead to the broadening of spectral emission peaks, the quality of the devices

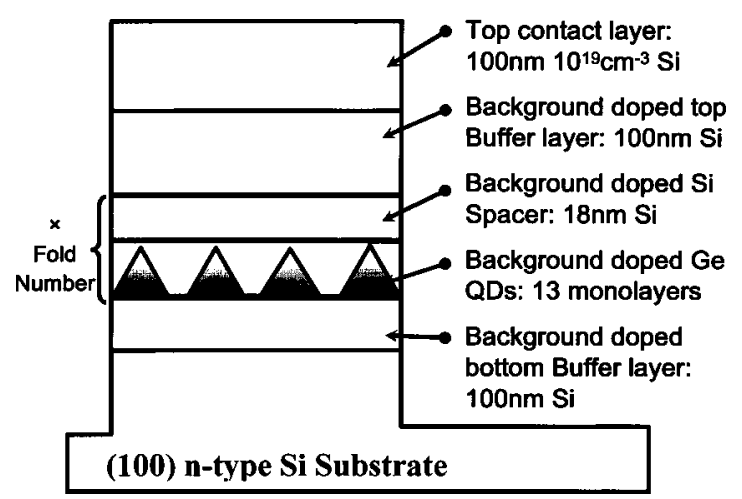

FIG. 1. Our sample structure (not to scale). The fold number is 5, 10, and 30 for our three QD LEDs. The background doping level calibrated by secondary ion mass spectroscopy was shown to be about $3 \times 10^{16} \mathrm{~cm}^{-3}$. 


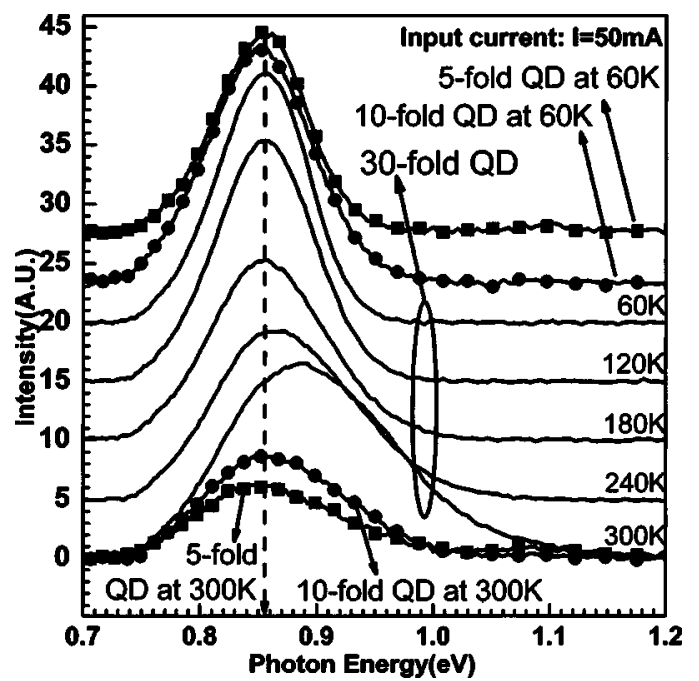

FIG. 2. EL spectra of 30-fold QD LED with different temperatures at the injection current of $50 \mathrm{~mA}$. The peak position at low temperature is shown by an arrow. At high temperature, the peak is shifted towards the higher energy, indicating the blueshift. The EL spectra of five- and ten-fold QD LEDs at $60 \mathrm{~K}$ are also plotted for a comparison.

relies on the possibility to control the size, shape, and uniformity of self-assembled QDs. With increasing the layer number, they nucleate closer to each other with almost unchanged dot size. When the dot spacing becomes smaller than $40 \mathrm{~nm}$, the dots merge to form a single dot, which almost covers the two underlying ones. The resulting base size is about $100 \mathrm{~nm}$. Once this large dot is formed, no further change in dot position is observed, and dots in consecutive layers nucleate exactly on top of each other. Moreover, their size assumes a stable value of $90 \mathrm{~nm}$, slightly less than the value of the initial merged dot. ${ }^{10}$ This merged dot leads to the controlled formation of many dots, which essentially do not interact, thus stabilize in size and spacing, and nucleate directly on top of each other. This approach to stack the QD layer is reported ${ }^{11}$ to improve the QD emission intensity.

The area of the mesa for our LEDs is $\sim 400 \times 400 \mu \mathrm{m}^{2}$. The aluminum (Al) metal was evaporated to act as a backside contact. On the other hand, to make a top transparent contact, a thin $(2 \mathrm{~nm}) \mathrm{Al}$ metal was deposited. Regarding the current-voltage characteristics, these diodes have $\sim 0.7 \mathrm{~V}$ for turn-on voltage and small leakage current of $\sim 100 \mathrm{nA}$ at the negative bias. The EL measurement was performed on these samples and detected by a large area InGaAs pin detector with standard lock-in technology.

The EL spectra of the Ge QD LEDs with the fold number of 5,10, and 30 at different temperatures are shown in Fig. 2. The peaks at around $0.85 \mathrm{eV}$ are originated from the $\mathrm{Ge}$ islands, while the usual $\mathrm{Si}-\mathrm{TO}$ peaks are located at $1.1 \mathrm{eV}$. To demonstrate the unusual evolution of EL spectra, we have sketched out the plot of variation of the EL peak positions as a function of temperature for five-, ten- and 30fold QDs as in Fig. 3. It is found that the position and shape of the EL spectra shown in Fig. 2 are almost the same for the three LEDs at $60 \mathrm{~K}$. But the five-fold QDs show slightly redshift due to the smaller recombination energy, ${ }^{11,12}$ while the 30-fold QDs show clearly the blue shift at $300 \mathrm{~K}$. The relationship between the experimental redshift of five-fold QDs agrees with the band-gap shrinkage as the temperature rises. It is noted that the ten-fold QDs have lower transition energy around $200 \mathrm{~K}$ and transfer to high energy at $250 \mathrm{~K}$. Downloaded 12 Feb 2009 to 140.112.113.225. Redistribution subject

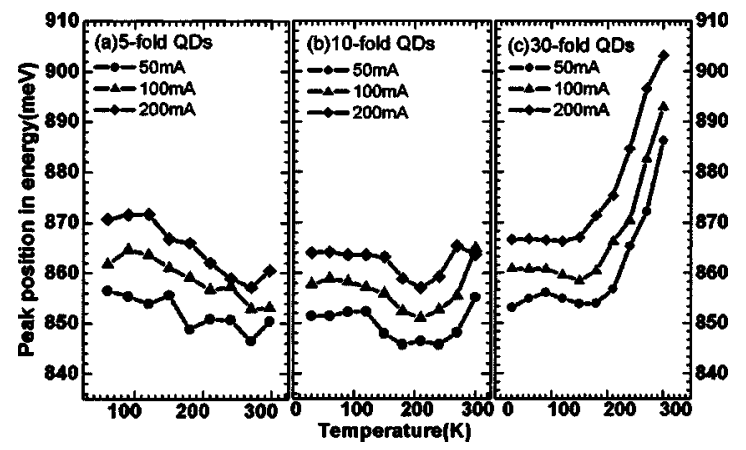

FIG. 3. Peak positions of EL spectra vs temperatures for (a) five-fold, (b) ten-fold, and (c) 30-fold QDs. The peak positions are the same at low temperature for all samples. At high temperature, five-fold QD shows the redshift while 30-fold QD shows the blueshift. The injected currents range from 50 to $200 \mathrm{~mA}$. The round, triangle, and diamond dot plotted curves stand for 50,100 , and $200 \mathrm{~mA}$, respectively.

Recently, M. Stoffel et al. ${ }^{13}$ have reported a similar experiment on ten-fold QDs, and have proposed an explanation that an increasing number of electrons starting to evaporate into the surrounding Si matrix would cause a blueshift of the EL signal with increasing the temperature. This behavior cannot explain the blueshift of our 30-fold QD because of the large shift of energy $\sim 30 \mathrm{meV}$.

Figure 4 shows the integral intensity of the spectra versus temperature. As represented by the solid triangles, the injection current increases from $50 \mathrm{~mA}-200 \mathrm{~mA}$ and the integral intensity tends to increase with temperature above $200 \mathrm{~K}$ for all the injection currents. The unusual enhancement of integral EL intensity is obvious for 30-fold QD LED, while the intensity of five-fold and ten-fold QD LEDs decreases gradually as represented by the circles and rectangle after the temperature of $200 \mathrm{~K}$. Consider the radiative region including depletion region, the hole and electron diffusion regions. By referring to the device structure as shown in Fig. 1 for our LEDs, the $p$-type region includes bottom $100 \mathrm{~nm}$ buffer layer, MQD layer, and top $100 \mathrm{~nm}$ buffer. Therefore the difference between five-fold, ten-fold, and 30-fold QD LEDs is the number of QDs outside depletion. If the diffusion length reaches longer to turn-on more QDs outside

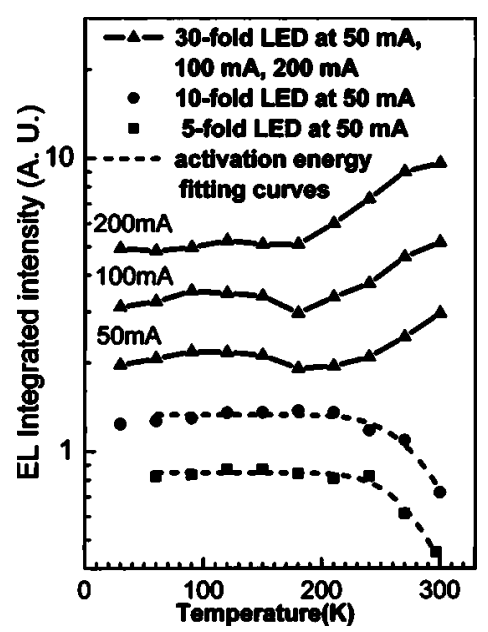

FIG. 4. Integral intensity of EL spectrum vs temperature for five-, ten-, and 30-fold QD LED at different injection current. The data for the five- and ten-fold LEDs under $50 \mathrm{~mA}$ injection current are also plotted for comparison. The dashed curves are fitted to estimate the activation energy. The activation energies are 263.6 and $256.8 \mathrm{meV}$ for five- and ten-fold QDs, respectively.

to AlP license or copyright; see http://apl.aip.org/apl/copyright.jsp 
depletion region, EL intensity may increase. As suggested in Ref. 14, the electron diffusion length for the pure Si low $p$-type region may increase with temperature at low current injection. Especially for $p$-type doping $N_{A}=2.4 \times 10^{17} \mathrm{~cm}^{-3}$, the diffusion length at $300 \mathrm{~K}$ is roughly 10 times that at $30 \mathrm{~K}$. At low temperature, the holes stay in the ground state of QDs. When temperature increases, some of the holes leave the ground state for the thermal energy. This phenomenon is indicated by the fact that the luminescence intensities shown in Fig. 4 for five-fold and ten-fold QDs are decreased with temperature. The dash lines for five-fold and ten-fold QDs are the fitting curves for activation energy. It calculated from the temperature dependence of integral intensity for five-fold and ten-fold QDs are found to be 263.6 and $256.8 \mathrm{meV}$, respectively. The sum of the activation energy and the QD band edge extracted from EL spectra at lowest temperature is around $1.1 \mathrm{eV}$ for the two samples. Because $\mathrm{Si}$ and $\mathrm{Ge}$ is of the type II band alignment, the activation energy is regarded as the hole confinement. As the integral intensity decreased, the holes that perform radiative recombination begin to leave the QD ground state at $\sim 200 \mathrm{~K}$. The hot carriers with sufficient energy to run over the barrier may perform nonradiative recombination. This implies that the probability for a nonradiative transition in Si barrier due to the defect is lager than that in each individual Ge QDs. For 30-fold QDs, the QD region is about $900 \mathrm{~nm}$. Therefore, the increment of the electron diffusion length with the temperature results in emitting more photons by capturing electrons in high fold QDs. Not only the integral intensity but also the blueshift in the electroluminescence spectra of the 30-fold QDs can be explained by our model as in the following.

Figure 5 shows the diameter and height of the MQDs of the 30-fold LED by the cross-section transmission electron micrographs. The QDs become large with increasing the fold number. When the fold number exceeds 5 , the dot size is intended to accordance. ${ }^{10}$ This phenomenon exists for all our samples. Compared with the initial layers of five-, ten-, and 30-fold QDs, the Si spacer layer keeps the similar shapes of QDs. The spacers of 30-fold QDs become especially contorted with the fold number over 15 . This phenomenon is attributed to the decrement of the strain of Si spacers surrounding the QDs. It also demonstrates that the MQDs system with higher fold number is trying to release the strain energy in some way. According to previous research, ${ }^{15}$ it suggests that the intermixing between QDs and Si spacer will be higher when more layers of QDs are grown. Since the strain induced intermixing results band-gap enlargement for the high-fold QDs, and the electron diffusion length intends to become longer for high temperature, EL spectra for 30fold QDs are shifted to blue for high temperature and their intensity also become large.

In conclusion, the electroluminescence of self-assembled Ge QDs is investigated as a function of fold number from 30 to $300 \mathrm{~K}$. The unusual behaviors for the 30-fold QD LED as compared with the five- and ten-fold ones are the blueshift and the integral intensity increment of the spectra with temperature. A possible explanation is raised that electrons diffuse far away at the high temperature due to the electron diffusion length increment with temperature. It enables more QD layers at the high-fold layer to emit photons. Those QDs have a large band gap due to the dot size and the intermixing effect of the Si and Ge. This may be a good way to achieve
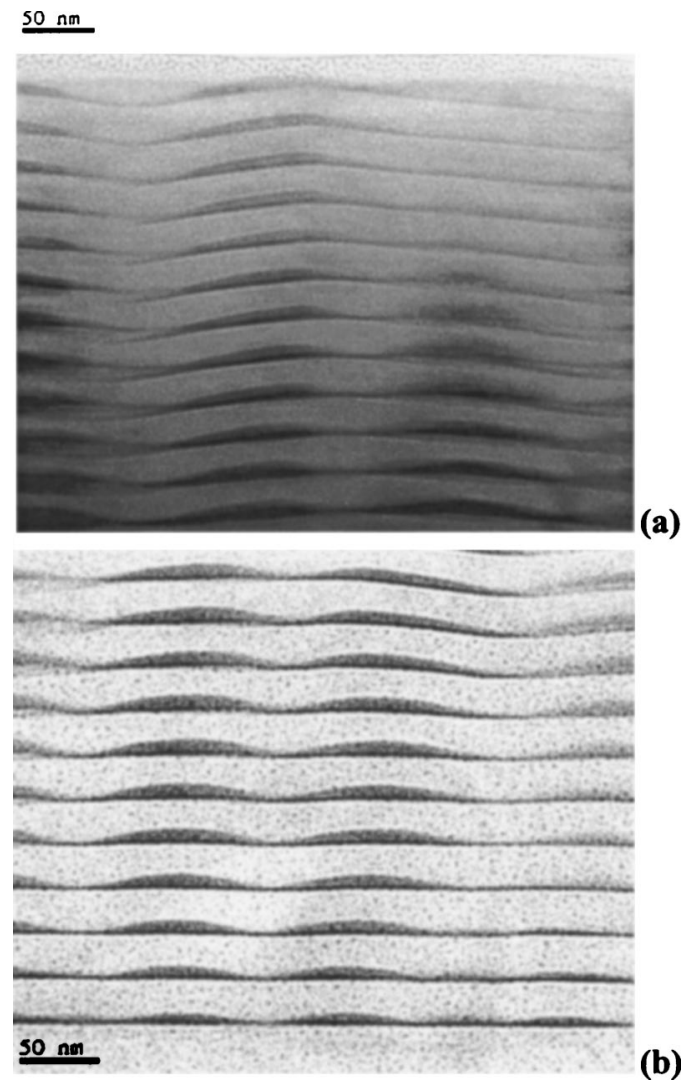

FIG. 5. TEM micrograph of 30-fold MQDs for the (a) final and (b) initial layers. The initial layers of the five- and ten-fold LEDs are similar with the 30-fold ones.

high luminescence intensities on $\mathrm{Ge} / \mathrm{Si}$ quantum dots for optical device applications.

This work was partly supported by the National Science Council of the Republic of China under Contract No. NSC 92-2215-E-002-022.

${ }^{1}$ J. C. Campbell, in Germanium Silicon: Physics and Materials: Optoelectronics in Silicon and Germanium Silicon, edited by R. Hull and J. C. Bean (Academic, San Diego, 1999), p. 347.

${ }^{2}$ T. Splett, K. Zinke, E. Petermann, H. Kasper, H.-J. Kibbel, and X. H. Herzog, IEEE Photonics Technol. Lett. 6, 59 (1994).

${ }^{3}$ G. Franzo, F. Priolo, S. Coffa, A. Polman, and A. Carnera, Appl. Phys. Lett. 64, 2235 (1994).

${ }^{4}$ R. Apetz, L. Vescan, A. Hartmann, C. Dieker, and H. Luth, Appl. Phys. Lett. 66, 445 (1995).

${ }^{5}$ L. Vescan, O. Chretien, T. Stoica, E. Mateeva, and A. Mück, Mater. Sci. Semicond. Process. 3, 383 (2000).

${ }^{6}$ T. Brunhes, P. Boucaud, S. Sauvage, F. Aniel, J.-M. Lourtioz C. Hernandez, Y. Campidelli, O. Kermarrec, D. Bensahel, G. Faini, and I. Sagnes, Appl. Phys. Lett. 77, 1822 (2000).

${ }^{7}$ K. Eberl, M. O. Lipinski, Y. M. Manz, W. Winter, N. Y. Jin-Phillipp, and O. G. Schmidt, Physica E (Amsterdam) 9, 164 (2001).

${ }^{8}$ E. W. Williams and R. Hall, Luminescence and the Light Emitting Diode (Pergamon, Oxford, 1978), p. 75.

${ }^{9}$ T. Takagahara and K. Takeda, Phys. Rev. B 46, 15578 (1992).

${ }^{10}$ E. Mateeva, P. Sutter, J. C. Bean, and M. G. Lagally, Appl. Phys. Lett. 71, 3233 (1997).

${ }^{11}$ L. Vescan, M. Goryll, K. Grimm, and C. Dieker, IEEE Topical Meeting on Silicon Integrated Circuits for RF Systems, Ann Arbor, MI, February, 1998, p. 38.

${ }^{12}$ L. Vescan, T. Stoica, O. Chretien, M. Goryll, E. Mateeva, and A. Muck, J. Appl. Phys. 87, 7275 (2000).

${ }^{13}$ M. Stoffel, U. Denker, and O. G. Schmidt, Appl. Phys. Lett. 82, 3236 (2003).

${ }^{14}$ D. B. M. Klaassen, Solid-State Electron. 35, 961 (1992).

${ }^{15}$ O. G. Schmidt and K. Eberl, Phys. Rev. B 61, 13721 (2000). 